\title{
1 Double nerve transfer to a single target muscle: experimental model in
}

\section{2 the upper extremity}

3 Matthias Luft ${ }^{1,2}$, Johanna Klepetko ${ }^{1,2}$, Silvia Muceli ${ }^{3}$, Jaime Ibáñez ${ }^{4,5}$, Vlad Tereshenko ${ }^{1,2}$,

4 Christopher Festin ${ }^{1,2}$, Gregor Längle ${ }^{1,2}$, Olga Politikou ${ }^{1,2}$, Udo Maierhofer ${ }^{1,2}$, Dario Farina ${ }^{4}$,

5 Oskar C. Aszmann ${ }^{1,6}$, Konstantin D. Bergmeister ${ }^{1,2,7}$

$6 \quad{ }^{1}$ Clinical Laboratory for Bionic Extremity Reconstruction, Department of Plastic, Reconstructive and

7 Aesthetic Surgery, Medical University of Vienna, Vienna, Austria

$8 \quad{ }^{2}$ Center for Biomedical Research, Medical University of Vienna, Vienna, Austria

$9 \quad{ }^{3}$ Department of Electrical Engineering, Chalmers University of Technology, Gothenburg, Sweden

$10 \quad{ }^{4}$ Department of Bioengineering, Imperial College London, London, UK

$11{ }^{5}$ Department of Clinical and Movement Neuroscience, University College London, London, UK

$12{ }^{6}$ Department of Plastic, Reconstructive and Aesthetic Surgery, Medical University of Vienna,

13 Vienna, Austria

$14{ }^{7}$ Karl Landsteiner University of Health Sciences, Department of Plastic, Aesthetic and Reconstructive

15 Surgery, University Hospital St. Poelten, Krems, Austria

$17 *$ Correspondence:

18 Konstantin Bergmeister, MD PhD

19 Clinical Laboratory for Bionic Extremity Reconstruction,

20 Department of Plastic, Reconstructive and Aesthetic Surgery

21 Medical University of Vienna

22 Waehringer Guertel 18-20

23 A-1090 Vienna, Austria

24 kbergmeister@gmail.com

25 Keywords: nerve injury, peripheral nerve, nerve transfer, targeted muscle reinnervation, rat model, microsurgery 
Abstract

29 Surgical nerve transfers are used to efficiently treat peripheral nerve injuries, neuromas, phantom

30 limb pain or improve bionic prosthetic control. Commonly, one donor nerve is transferred to one

31 target muscle. However, the transfer of multiple nerves onto a single target muscle may increase the

32 number of muscle signals for myoelectric prosthetic control and facilitate the treatment of multiple

33 neuromas. Currently, no experimental models are available for multiple nerve transfers to a common

34 target muscle in the upper extremity. This study describes a novel experimental model to investigate

35 the neurophysiological effects of peripheral double nerve transfers. For this purpose, we developed a

36 forelimb model to enable tension-free transfer of one or two donor nerves in the upper extremity.

37 Anatomic dissections were performed to design the double nerve transfer model $(\mathrm{n}=8)$. In 62 male

38 Sprague-Dawley rats the ulnar nerve of the antebrachium alone $(n=30)$ or together with the anterior

39 interosseus nerve $(n=32)$ was transferred to reinnervate the long head of the biceps brachii. Before

40 neurotization, the motor branch to the biceps' long head was transected at the motor entry point and

41 resected up to its original branch to prevent auto-reinnervation. In all animals, coaptation of both

42 nerves to the motor entry point could be performed tension-free. Mean duration of the procedure was

$4349 \pm 13 \mathrm{~min}$ for the single nerve transfer and $78 \pm 20 \mathrm{~min}$ for the double nerve transfer. Twelve

44 weeks after surgery, muscle response to neurotomy, behavioral testing, retrograde labeling and

45 structural analyses were performed to assess reinnervation. These analyses indicated that all nerves

46 successfully reinnervated the target muscle. No aberrant reinnervation was observed by the originally

47 innervating nerve. Our observations suggest a minimal burden for the animal with no signs of

48 functional deficit in daily activities or auto-mutilation in both procedures. Furthermore, standard

49 neurophysiological analyses for nerve and muscle regeneration were applicable. This newly

50 developed nerve transfer model allows for the reliable and standardized investigation of neural and

51 functional changes following the transfer of multiple donor nerves to one target muscle. 


\section{$1 \quad$ Introduction}

60 Nerve transfers offer a variety of therapeutic possibilities in modern extremity reconstruction, such as

61 treating peripheral nerve injuries, neuromas, phantom limb pain, improving prosthetic control or

62 restoring function following spinal cord injuries (Aszmann et al., 2015;Farina et al., 2017;Dumanian

63 et al., 2019; Van Zyl et al., 2019). Compared to conventional nerve repair modalities, nerve transfers

64 are capable of bypassing slow peripheral nerve regeneration (Terzis and Papakonstantinou, 2000),

65 thus preventing irreversible muscle fibrosis before reinnervation (Mackinnon and Novak, 1999). For

66 this purpose, nearby nerves with a sufficient axonal load and lesser functional importance are

67 neurotomized and transferred to the injured nerve (Oberlin et al., 1994;Bertelli et al., 1997). Because

68 of overall faster regeneration and better functional outcomes compared to nerve grafting, this surgical

69 procedure has been able to improve the devastating effects of peripheral nerve and brachial plexus

70 lesions, which have otherwise often led to long-term health impairment and subsequent socioeconomic costs (Mackinnon and Novak, 1999;Terzis and Papakonstantinou, 2000;Bergmeister et al., 2020). Additionally, they are used in a procedure termed targeted muscle reinnervation (TMR)

to improve myoelectric prosthetic control (Kuiken et al., 2009; Kapelner et al., 2016), treat neuromas

or phantom limb pain (Mioton et al., 2020). Here, amputated nerves within an extremity stump are

transferred to residual stump muscles, thus significantly improving the recording of neural activity about motor intent and the control of myoelectric prostheses. Generally, one donor nerve is

transferred to one target muscle head and this concept has been well studied with high clinical success (Kuiken et al., 2009;Aszmann et al., 2015;Farina et al., 2017). However, the use of multiple nerve transfers to a single target muscle head may provide additional benefits for these clinical indications but has not been clinically explored. Although several nerve transfer models have been

81 established (Kuiken et al., 1995;Bergmeister et al., 2016;Aman et al., 2019), none of them has

82 investigated multiple peripheral nerve transfers in the upper extremity. Only one model where 83 multiple donor nerves are used to restore muscle function in the rat hindlimb has been described 
84 (Kuiken et al., 1995). However, as most nerve injuries occur in the upper extremity, an upper

85 extremity model for experimental investigation of this concept is needed (Scholz et al., 2009).

86 In this study, we propose a surgical nerve transfer model to allow the transfer of multiple donor

87 nerves to a single muscle head and we validate this model in the rat forelimb. This model allows for

88 reliable analyses with all standard neurophysiological investigations of the motor unit for possible

89 implementation of this concept to clinical application.

\section{$90 \quad 2 \quad$ Materials and methods}

\section{$91 \quad 2.1 \quad$ Experimental design}

92 Eight rat cadavers were dissected to design the double nerve transfer procedure. An important

93 criterion for the selection of the donor nerves and the target muscle was clinical relevance. First,

94 eligible peripheral motor nerves were determined for a reliable, tension-free transfer to the long head

95 of the biceps muscle. Then, the topographical relationships between the biceps' long head, its motor

96 nerve branch, the ulnar nerve in the antebrachium (UN) and the anterior interosseus nerve (AIN)

97 were studied and subsequently compared to the human anatomy. These studies verified the

98 anatomical feasibility of transferring both the distal UN and AIN to the long head of the biceps.

99 Sixty-two Sprague-Dawley rats aged 8-10 weeks were randomly allocated into two groups by an

100 animal care taker to investigate functional and structural changes following single (SNT) and double

101 nerve transfer (DNT). Thirty-two animals were assigned to the DNT group (Figure 1), while 30

102 animals underwent the single nerve transfer of the UN and were used as control (Figure 1). Twelve

103 weeks after surgery, microscopic inspection of the motor entry point $(n=62)$, nerve crush and

104 neurotomy ( $\mathrm{n}=32)$, and Terzis' grooming test $(\mathrm{n}=51)$ (Inciong et al., 2000) were performed. After the

105 final functional assessments, muscle specimens were harvested and weighed $(n=32)$. Thirty-eight

106 animals were assigned for retrograde labeling analyses. Sample size calculations performed by a 
107 biostatistician were considered in the planning of the studies. Planning, conducting and reporting of

108 experiments were performed according to the ARRIVE (Animal Research: Reporting of In Vivo

109 Experiments) guidelines (Percie Du Sert et al., 2020). The protocols for these experiments were

110 approved by the ethics committee of the Medical University of Vienna and the Austrian Ministry for

111 Research and Science (reference number BMBWF- 66.009/0413-V/3b/2019) and strictly followed

112 the principles of laboratory animal care as recommended by the Federation of European Laboratory

113 Animal Science Associations (FELASA)(Guillen, 2012).

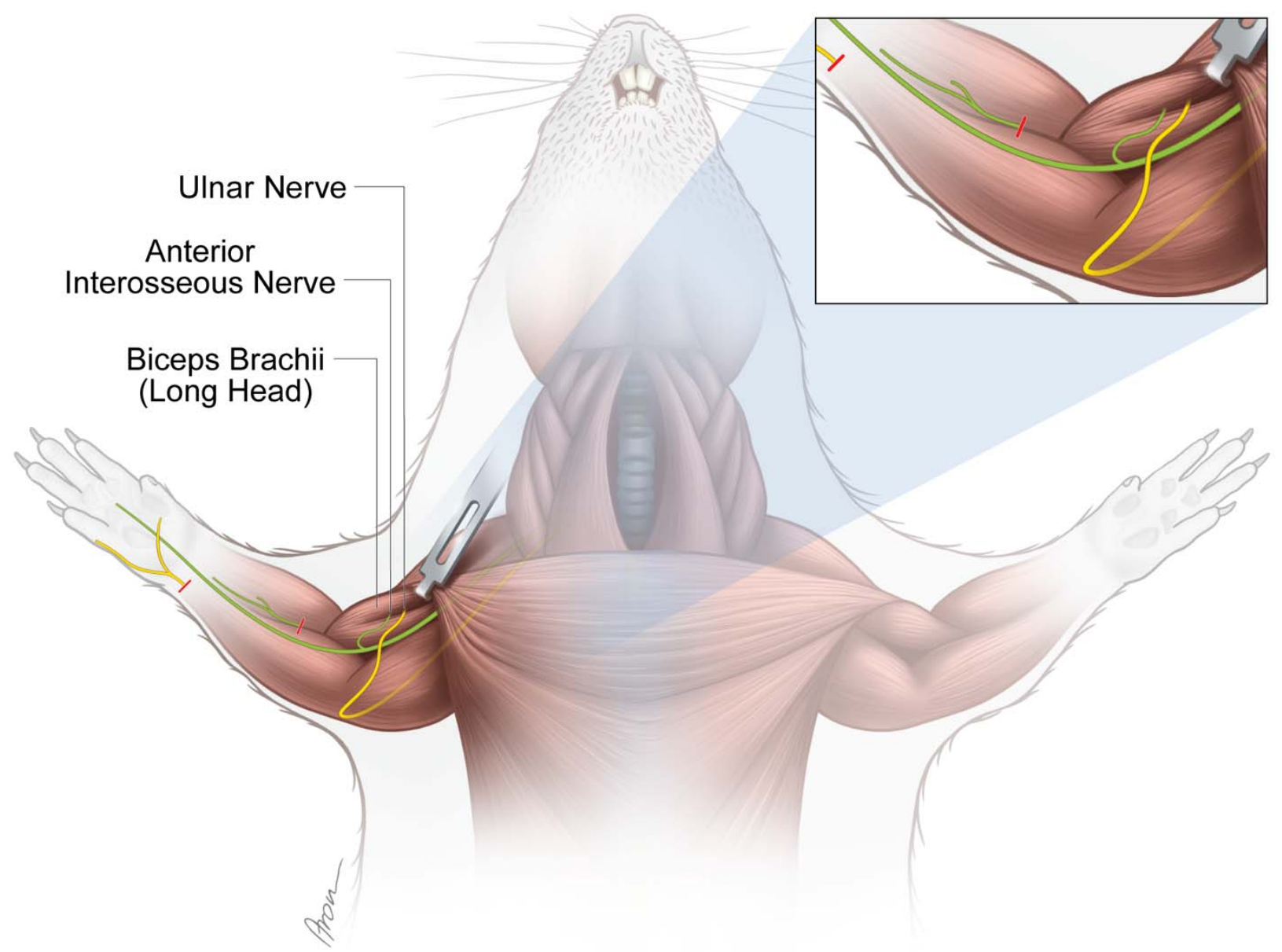

115 Figure 1. Experimental nerve transfer models. Single-nerve transfer model: The UN (yellow) was transected distally

116 to the palmar cutaneous branch in the forearm and surgically transferred to reinnervate the long head of the biceps ( $\mathrm{n}=30$ ).

117 Multiple-nerve transfer model: Both the UN (yellow) and AIN (green) were redirected to reinnervate the long head of the

118 biceps $(\mathrm{n}=32)$. Before both nerve transfer procedures, the originally innervating branch of the MCN was removed. The 
119 untreated contralateral biceps muscles served as internal control for both groups. The red lines indicate the level of

120 transection. Credit: Aron Cserveny.

\section{$121 \quad 2.2 \quad$ Nerve transfer model}

122 For each procedure, anesthesia was induced with ketamine $(100 \mathrm{mg} / \mathrm{kg})$ and xylazine $(5 \mathrm{mg} / \mathrm{kg})$

123 intraperitoneally and maintained by volume-controlled ventilation $(40 \%$ O2, room air, $1.5-2 \%$

124 isoflurane) following orotracheal intubation. Piritramide $(0.3 \mathrm{mg} / \mathrm{kg})$ was administered

125 subcutaneously for analgesia. Furthermore, the drinking water was mixed with piritramide and

126 glucose (30 mg piritramide and $30 \mathrm{ml} 10 \%$ glucose dissolved in $250 \mathrm{ml}$ drinking water) and

127 administered ad libitum for pain relief during the first seven postoperative days. After the

128 experimental tests, animals were euthanized with a lethal dose of pentobarbital (300 mg/kg) injected

129 intracardially under deep anesthesia. All animals were examined daily by an animal keeper for pain,

130 sensory deficits, impairments in daily activities, wound dehiscence and infection. All nerve transfer

131 procedures were performed by the same surgeon and assistant.

\section{$132 \quad$ 2.2.1 Single nerve transfer}

133 A lazy S-shaped incision was made from $5 \mathrm{~mm}$ caudal to the greater tubercle of the humerus over the

134 medial epicondyle along the ulnar side of the forearm until $5 \mathrm{~mm}$ proximal to the forepaw (Figure

135 2A). Following the dissection of the subcutaneous tissue, the antebrachial fascia was opened through

136 an incision placed over the palmaris longus muscle to preserve the underlying ulnar collateral vessels.

137 Then, the flexor carpi ulnaris muscle was bluntly mobilized and retracted ulnarly using a Magnetic

138 Fixator Retraction System (Fine Science Tools, Heidelberg, Germany) to expose the UN. Further

139 exposure of the dorsal and palmar cutaneous branches of the UN was carried out using an operating

140 microscope (Carl Zeiss, Munich, Germany) (Figure 2B). The palmar branch was cut right after its

141 emergence and the UN was subsequently transected as distally as possible. The UN was dissected

142 proximally to its distal exit from the cubital tunnel while preserving the ulnar artery and basilic vein. 
143 Intraneural dissection allowed for conservation of the dorsal cutaneous and flexor carpi ulnaris motor

144 branches (Figure 2B), while facilitating a tension-free nerve coaptation. Next, the incision of the

145 antebrachial fascia was extended proximally to open the brachial fascia above the cubital fossa and

146 biceps. Subsequently, the pectoral muscles were retracted to expose the musculocutaneous nerve's

147 (MCN) branch to the long head of the biceps running along the bicipital groove (Figure 2C). The

148 motor branch of the MCN to the biceps' long head was then cut at the motor insertion point and the

149 proximal segment subsequently removed from its division to prevent spontaneous regeneration. Next,

150 the UN was routed proximally over the cubital fossa and coapted tension-free to the epimysium near

151 the original motor insertion point with one 11-0 (Ethilon, Ethicon, Johnson \& Johnson Medical Care,

152 USA) simple interrupted stitch (Figure 2D). 

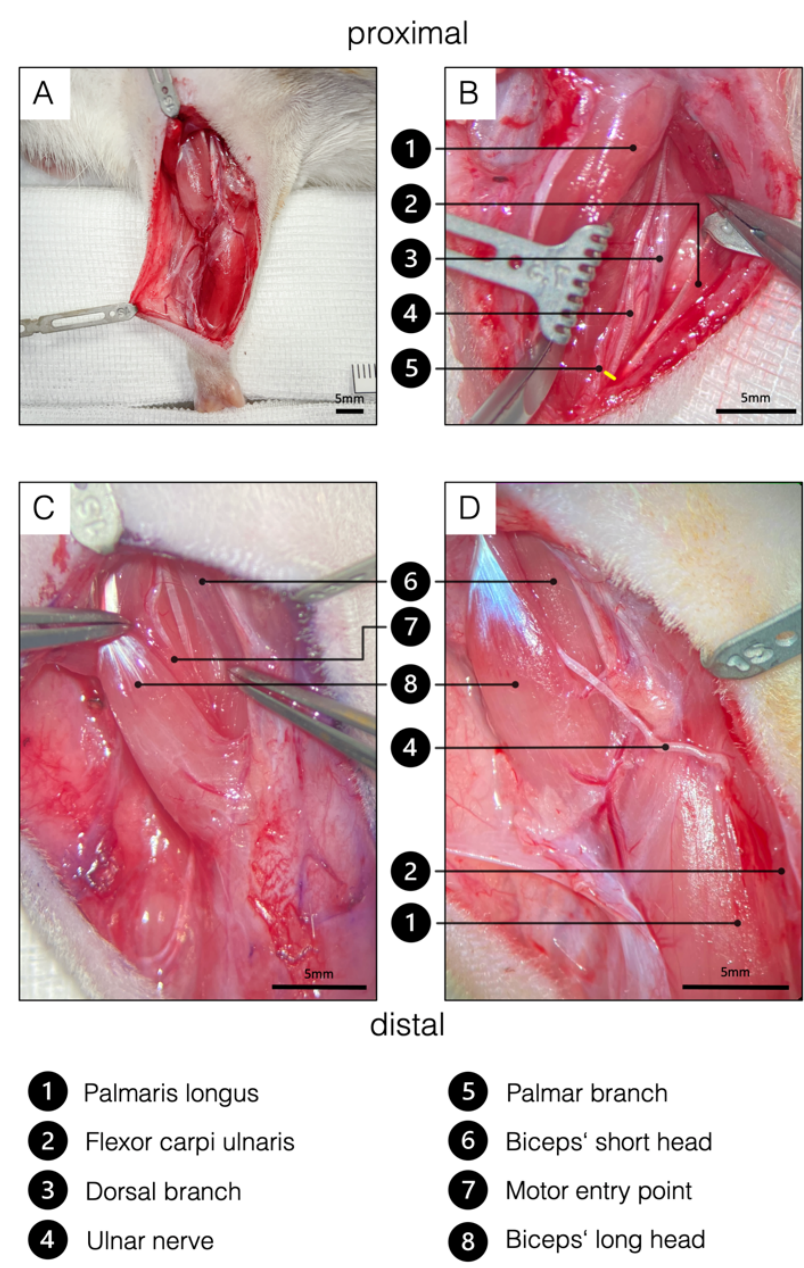

154 Figure 2. Surgical procedure of the ulnar nerve transfer. (A) Overview of the rats' supinated right forelimb after the

155 brachial and antebrachial fascia were removed. (B) Two blunt retractors have been placed to pull the flexor carpi ulnaris 156 and the palmaris longus apart, revealing the underlying UN. The yellow line indicates the level of transection to gain sufficient length to reach the biceps' long head tension-free. To achieve this, the palmar cutaneous branch must be transected, while the dorsal cutaneous branch can be preserved. (C) For better visualization, the brachial fascia was opened above the biceps. A sharp retractor was placed to pull back the pectoral muscles and thus revealed the two biceps heads, which were bluntly separated. In the deep bicipital groove, the MCN and its motor branch to the long head of the

161 biceps were identified. Maximum length of the motor branch to the long head was removed to prevent spontaneous 162 regeneration. (D) Eventually, the UN was rerouted from between the palmaris longus and flexor carpi ulnaris to the long 163 head of the biceps and sutured to the epimysium at the former original motor entry point. This procedure on the one hand 164 spares the denervation of the flexor carpi ulnaris and the flexor digitorum superficialis and the invasive dissection through 165 the cubital tunnel. 


\subsubsection{Double nerve transfer}

167 The skin incision, exposure of the distal UN as well as the denervation of the biceps' long head were

168 performed as described in the single nerve transfer. Before coaptation of the UN, the median nerve

169 and AIN were dissected. For better exposure of the AIN, one blunt retractor was carefully placed to

170 pull the proximal belly of the pronator teres muscle ulnarly (Figure 3A). After identifying the AIN, it

171 was transected and dissected proximally in an intraneural fashion to its branching point (Figure 3A).

172 Then, both the UN and the AIN were neurotized to the epimysium near the original motor insertion

173 point with one 11-0 (Ethilon, Ethicon, Johnson \& Johnson Medical Care) simple interrupted stitch

174 each (Figure 3B). Significant caliber differences between the motor branch of the biceps' long head

175 and the two transferred nerves required neurotization directly to the epimysium. In this way, the

176 regeneration distance was kept as short as possible, hence minimizing the reinnervation time. It is

177 particularly important not to place the two nerves in direct proximity in the tissue (Figure 3B) as this

178 increases the complexity of the dissection and therefore the risk of injuring the nerves in the follow-

179 up examinations. Wound closure was performed with fascial and deep dermal 6-0 (Vicryl, Ethicon,

180 Johnson and Johnson Medical Care, Austria) simple interrupted sutures followed by running

181 subcuticular suture with 6-0 (Vicryl, Ethicon, Johnson and Johnson Medical Care, Austria). 


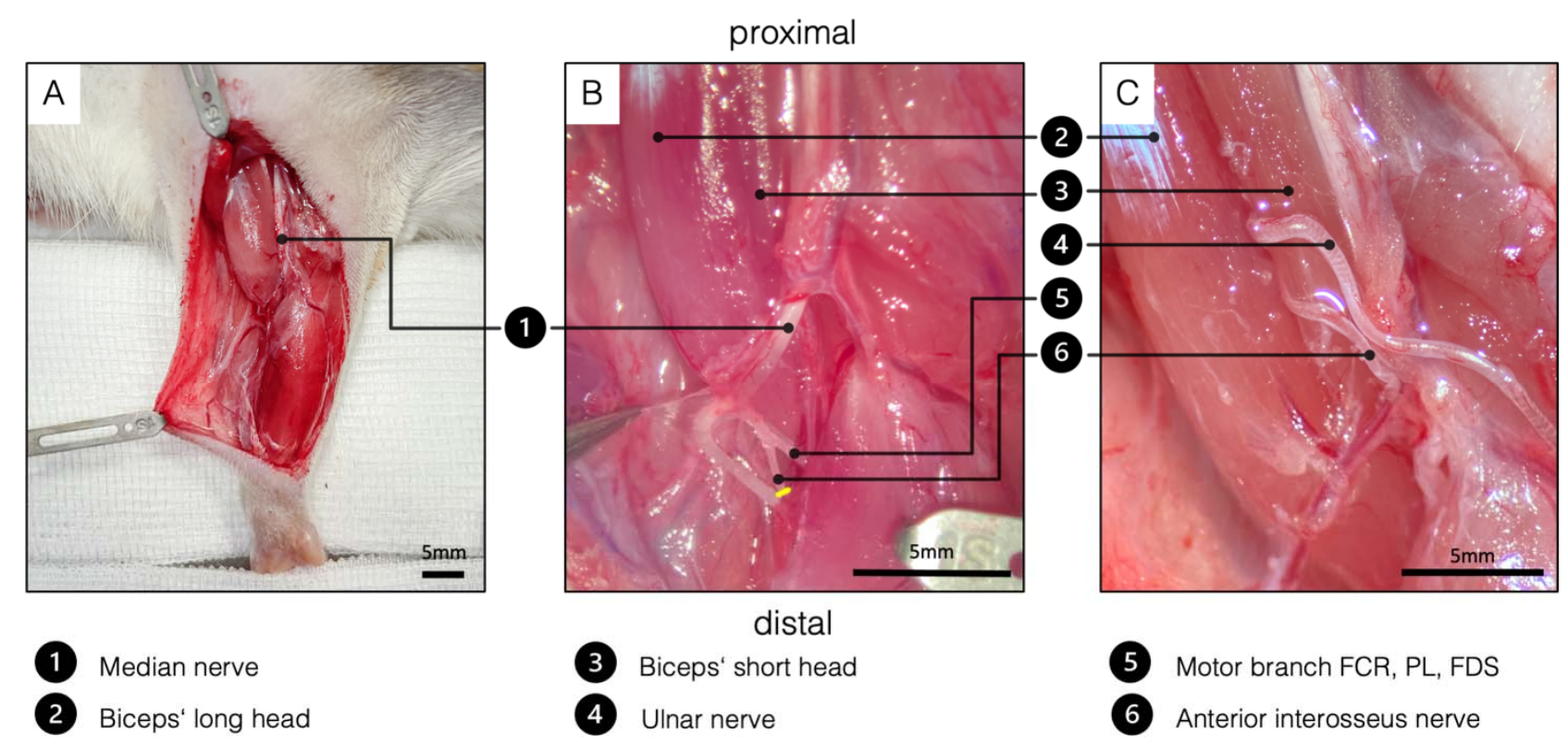

183 Figure 3. Surgical procedure of the double nerve transfer. (A) General view of the right supinated forelimb. The

184 proximal hook pulls the pectoral muscles towards proximal for better presentation. (B) The brachial and antebrachial

185 fascia and the motor branch to the pronator teres muscle were removed for better visualization. In the cubital fossa, three

186 branches arise from the median nerve: one muscle branch supplying the pronator teres (resected), one muscle branch

187 supplying the flexor carpi radialis, palmaris longus and flexor digitorum superficialis and the AIN supplying pronator

188 quadratus, flexor pollicis longus and flexor digitorum profundus. After transecting the AIN (yellow line), proximal

189 dissection in an intraneural fashion gains sufficient length to reach the biceps' motor entry point. (C) Surgical site before

190 wound closure, after both the UN and the AIN were transferred to the physiological motor entry point of the long head of

191 the biceps. (FCR - flexor carpi radialis. PL - palmaris longus. FDS - flexor digitorum superficialis).

\section{$192 \quad 2.3 \quad$ Behavioral evaluation}

193 Quantitative assessment of grooming behavior was carried out and filmed twelve weeks after the

194 single $(\mathrm{n}=21)$ and double nerve transfer $(\mathrm{n}=30)$ using Terzis' grooming test (Inciong et al., 2000), a

195 modification of Bertelli's grooming test (Bertelli and Mira, 1993). To keep the animals' stress level

196 at a minimum, testing was performed in the animals' familiar environment. In brief, 1 to $3 \mathrm{ml}$ of

197 water was sprinkled on the rats' snouts, which led to consistent bilateral grooming movements of the

198 forelimbs. Grading of the grooming performance was assessed by the following score: grade 1, paws 
reach mouth or elbow is extended; grade 2, paws reach mouth and beneath eyes; grade 3, paws reach

200 eyes; grade 4, paws reach between eyes and ears; grade 5, paws reach behind the ears. The slow-

201 motion video sequences were graded by a blinded observer.

\section{$202 \quad 2.4 \quad$ Retrograde labeling}

203 Assessment of the motor unit at the spinal cord level after nerve transfer surgery was performed via

204 retrograde labeling as previously described (Hayashi et al., 2007). In brief, retrograde tracers are

205 taken up by terminal axons and transported via retrograde axonal transport to label the cell somas in

206 the spinal cords' ventral root. In eight additional untreated control animals both the UN in the

207 antebrachium and the AIN were transected and placed into conduit reservoirs for one hour, either

208 filled with $5 \mu 1$ of $10 \%$ Fluoro-Ruby (Invitrogen, Carlsbad, CA, USA) or $5 \mu 1$ of $2 \%$ Fast-Blue

209 (Polysciences, Warrington, PA, USA). Tracer leakage was prevented by sealing the reservoir around

210 the nerve with Vaseline (Vaselinum album, Fagron, Glinde, Germany). Hence, the corresponding

211 motor neuron pools in the spinal cord (C8-Th1) were localized (Figure 4). To further prevent bias

212 due to differences in penetration of the tracers, the nerves were alternately colored with Fluoro-Ruby

213 and Fast-Blue. Additionally, twelve weeks following the SNT ( $\mathrm{n}=15)$ and DNT ( $\mathrm{n}=15)$ surgery,

214 motor neurons reinnervating the long head of the biceps were studied. Through a $15 \mathrm{~mm}$ incision

215 above the biceps, the biceps' long head and its insertion site were exposed. A Hamilton micro syringe

216 was then used to inject $10 \mu 12 \%$ Fluoro-Gold (Fluorochrome, LLC, Denver, CO) evenly into the

217 biceps' long head near the motor insertion site. After tracer injection with a small gauge needle, the

218 syringe was kept inside the muscle for one minute before slowly withdrawing it to keep leakage to a

219 minimum. Seven days following retrograde labeling, the animals were deeply anesthetized by a lethal

220 dose of xylazine, ketamine and pentobarbital intraperitoneally before the left ventricle was perfused

221 with $400 \mathrm{ml}$ of $0.9 \% \mathrm{NaCl}$ followed by $400 \mathrm{ml}$ of $4 \%$ paraformaldehyde (PFA) solution. Then, the

222 spinal cord segments C4-Th2 were harvested and stored in $4 \%$ PFA for 24 hours at $+4^{\circ}$, followed by 


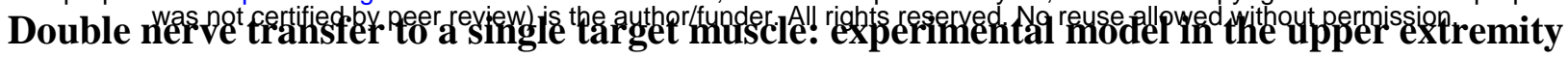

24h in $0.1 \mathrm{M}$ phosphate buffered saline PBS at $+4^{\circ}$. Then, the specimens were dehydrated in a PBS

224 solution with increasing sucrose concentrations of $10 \%, 25 \%$ and $40 \%$ for 24 hours each before

225 embedding them in Tissue-Tek ${ }^{\circledR}$ O.C.T. ${ }^{\text {TM }}$ Compound (Sakura Finetek Europe B.V., Alphen aan den

226 Rijn, Netherlands). Spinal cord segments were cut longitudinally into $40-\mu \mathrm{m}$ sections using a cryostat

227 (Leica, Germany). To assess the reinnervation, each spinal cord section was analyzed in an observer

228 blinded setting using a fluorescence microscope (Carl Zeiss, Munich, Germany). Spinal cord

229 segments of labeled motor neurons after DNT (Fluoro-Gold) were compared to the double labeled

230 (Fast-Blue, Fluoro-Ruby) segments of the untreated animals.

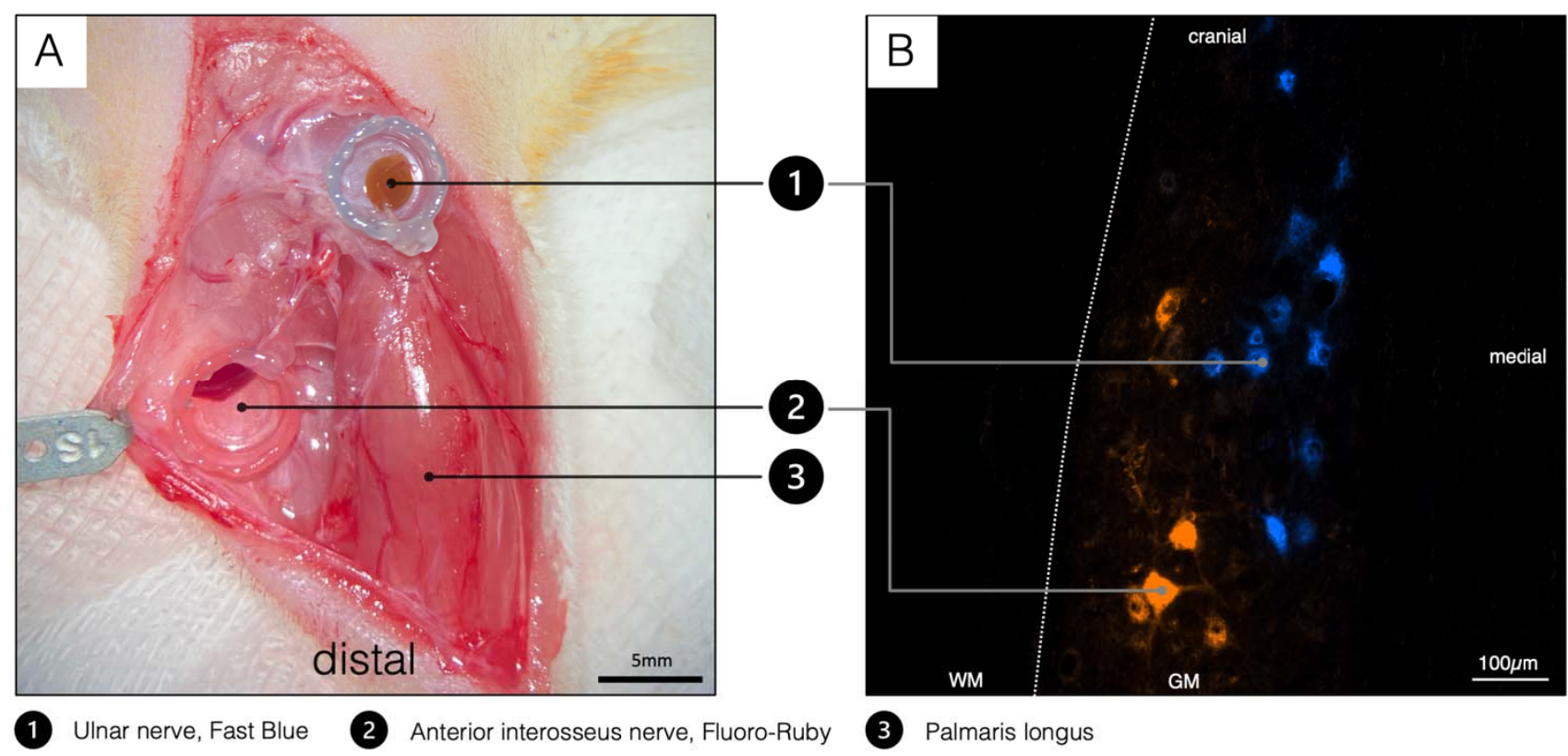

232 Figure 4. Double retrograde labeling. (A) The selected donor nerves were both dissected in a right forelimb and placed

233 in a conduit reservoir filled with Fast-Blue (UN) and Fluoro-Ruby (AIN) respectively for one hour. Wet sterile swabs

234 were placed above the surgical site to prevent the tissue from drying and the fluorescent dyes from bleaching.

235 (B) Spinal cord section C8-Th1. Labeled AIN (orange) and UN motoneuron pool (blue).

236 WM - white matter, GM - grey matter.

\subsection{Neuromuscular analyses}

238 The lengths of both the UN (n=6) and AIN ( $\mathrm{n}=6)$ were measured intraoperatively before coaptation

239 to the muscle. Twelve weeks following surgery, the motor entry point was microscopically examined 
240 for proper reinnervation and neuroma formation in all animals. Muscle reaction to nerve crush (see

241 Video 2 for muscle reaction to MCN crush in the control side) and neurotomy was assessed in

242 animals following DNT ( $\mathrm{n}=17)$ and compared to animals following SNT ( $\mathrm{n}=15)$. For internal control,

243 the motor branches to the biceps' long head were crushed and neurotomized in the contralateral

244 forelimbs. Conclusively, to assess neuromuscular regeneration after denervation, the biceps muscles

245 were resected and weighed immediately after removal using a microscale.

\section{$246 \quad 2.6 \quad$ Statistical analysis}

247 An ANCOVA was conducted to determine effects of the nerve transfer procedure (SNT and DNT) on

248 the reinnervated muscle mass after adjusting for control muscle mass. In addition, a paired-samples

249 t-test was used to determine whether there was a change of muscle mass following SNT or DNT

250 between the two sides. All data analyses were performed using SPSS Statistics for Macintosh,

251 Version 25.0 (IBM, Armonk, New York, USA).

\section{$252 \quad 3 \quad$ Results}

\section{$253 \quad 3.1 \quad$ Nerve transfer surgery}

254 All animals survived the surgical nerve transfers and showed normal gait and grasping behavior in

255 the twelve-week follow-up period. All animals were able to carry out activities of daily behavior

256 unhindered and no signs of severe pain, wound dehiscence, auto-mutilation or infection were

257 documented. Mean surgery time was $49 \pm 13 \mathrm{~min}$ for the SNT procedures and $78 \pm 20 \mathrm{~min}$ for the 258 DNT procedures.

\section{$259 \quad 3.2$ Behavioral evaluation}


260 Slow motion video sequence analysis by a blinded evaluator showed that twelve weeks following the

261 SNT and DNT, all animals could consistently reach behind their ears and therefore achieved a

262 maximum score of 5 (Video 1).

\section{$263 \quad 3.3 \quad$ Retrograde Labeling}

264 Analyses of the spinal cord following UN transfer showed adequate motor neuron staining in the

265 corresponding segments (Th1-C8). When comparing the spinal cords of the untreated animals with 266 spinal cords of animals which underwent DNT, the distribution pattern of the longitudinally arranged

267 Fluoro-Gold dyed clusters provides strong evidence that both the UN and AIN innervated the biceps'

268 long head (see Figure 4 for a representative example). Furthermore, no signs of spontaneous

269 regeneration from the MCN were noted by analyzing the corresponding spinal cord segments (C5-

270 C7).

\subsection{Neuromuscular analyses}

272 Both the donor nerve branches, and biceps' motor entry point were topographically consistent. The

273 UN measured a mean length of $23.08 \pm 1.36 \mathrm{~mm}$ from the distal exit of the cubital tunnel to the distal

274 stump. The AIN transfer provided a mean length of $10.50 \pm 1.61 \mathrm{~mm}$ measured from its branching

275 off the median nerve to the distal stump.

276 Twelve weeks following nerve transfer surgeries, macroscopic examination of all biceps motor entry

277 points showed successful reinnervation but no auto-innervation by the MCN and no signs of neuroma

278 were detected. Adequate muscle fibrillation was observed in all animals upon crushing and 279 neurotomizing the donor nerves individually following SNT and DNT (UN crush and AIN crush 280 response is shown in video 3 and 4 respectively). 


\subsubsection{Comparison of reinnervated muscle mass}

282

283

284

285

286

287

288

289

290

291

292

293

294

295

296

297

298

299

300

301

302

303

There was a linear relationship between treated and untreated muscle mass for each nerve transfer procedure, as assessed by visual inspection of a scatterplot. There was homogeneity of regression slopes as the interaction term was not statistically significant, $F(1,28)=.238, p=.630$. Standardized residuals for the interventions and for the overall model were normally distributed, as assessed by Shapiro-Wilk's test $(p>.05)$. There was homoscedasticity and homogeneity of variances, as assessed by visual inspection of a scatterplot and Levene's test of homogeneity of variance $(p=.504)$, respectively. There were no outliers in the data, as no cases were detected with standardized residuals greater than \pm 3 standard deviations.

After adjustment for control muscle mass, there was a statistically significant difference in muscle mass between the treated sides following SNT and DNT, $F(1,29)=24.030$, *** $p<.001$, partial $\eta^{2}=$ .453. Muscle mass was statistically significantly larger in the DNT group $(303.01 \pm 7.76 \mathrm{mg})$ compared to the SNT group $(245.57 \pm 8.29 \mathrm{mg})$, with a mean difference of 57.45 (95\% CI, 33.48 to 81.41) $\mathrm{mg}, * * * p<.001$. Data are reported adjusted mean \pm standard error.

\subsubsection{Comparison of reinnervated and control muscle mass}

No outliers were detected as assessed by inspection of a boxplot. The assumption of normality was not violated, as assessed by Shapiro-Wilk's test for the SNT $(p=.758)$ and DNT group $(p=.307)$.

The mean muscle mass was reduced following SNT $(235.07 \pm 44.05 \mathrm{mg})$ as opposed to the untreated contralateral side $(292.93 \pm 35.17 \mathrm{mg})$ with a statistically significant decrease of -57.87 (95\% CI, 77.38 to -38.35$) \mathrm{mg}, t(14)=-6.360,{ }^{* * *} p<.001, d=1.64$. However, mean muscle mass following DNT $(312.28 \pm 37.74 \mathrm{mg})$ compared to the untreated contralateral side $(315.97 \pm 28.22 \mathrm{mg})$ was similar and showed no statistically significant change $(p=.571)$. Data are reported as mean \pm standard deviation. 
The present study provides a robust and easily accessible model for surgical double nerve transfers to a single target muscle in the rat's upper extremity. We offer detailed step-by-step instructions on how to reproduce this model, including potential pitfalls. For comparison, the model also offers a

308 description of a single nerve transfer to the same target muscle. We employed nerve crush, 309 neurotomy, behavioral analysis and retrograde labeling which indicated that neuromuscular 310 regeneration of two donor nerves occurred into one target muscle.

311 To our knowledge, only one rat model for multiple peripheral innervation of a single target has been

312 described. However, that previous model was for the lower extremity and did not provide detailed

313 description for step by step reproduction of the model (Kuiken et al., 1995). Hindlimb models do not

314 adequately represent the physiology of upper extremity nerve transfers and targeted muscle

315 reinnervation procedures. This notion is supported by the clinical discrepancy between the excellent

316 outcomes for upper extremity compared to the poor outcomes for lower extremity nerve transfers

317 (Ray et al., 2016). Furthermore, most nerve transfers are currently conducted in the upper extremity

318 for both nerve reconstruction and prosthetic control. We already established single peripheral nerve

319 transfer models in the upper extremity (Bergmeister et al., 2016;Aman et al., 2019), which were

320 considered for developing this novel model. For this purpose, we conducted anatomical dissections in

321 eight rat cadavers to design the DNT concept to allow tension-free approximation of the two motor

322 nerves to the target biceps muscle. Theoretically, many other target muscles are also feasible due to

323 the sufficient length of both the UN and AIN. However, the biceps muscle provides an optimal target

324 that is accessible for all standard structural and functional analyses and accurately represents a

325 surgical target in clinical nerve transfer scenarios as well.

326 The implementation of this model requires an operating microscope, a set of microsurgery tools and

327 advanced microsurgical skills to achieve reproducible results. In our experience, dissection of the UN

328 in the antebrachium can be performed in a straightforward manner and preservation of the motor

329 branch to the flexor carpi ulnaris muscle, the dorsal sensory branch and the ulnar artery is easily 
330 feasible. Subsequently, transecting the UN as distally as possible allows for tension-free coaptation to

331 the proximal target muscle. Exposure of the MCN's motor branch to the long head of the biceps is

332 best achieved in the bicipital groove by retracting the overlaying pectoral muscles medially. Here,

333 considerable care must be taken when dividing the two bicep heads to preserve the bicipital artery,

334 which enters the long head in the distal portion and advances in proximal direction. Injury to this

335 vessel has shown to affect functional measures in previous experiments. Another hazard in the DNT

336 model is potential injury of the median vessels in the cubital fossa. To prevent this scenario, special

337 attention is required during the dissection of the median nerve, because the median vessels are either

338 found directly beneath or above the nerve. It is mandatory to dissect the AIN intraneurally to its

339 proximal branching point to enable tension-free coaptation to the original motor point of the biceps.

340 Due to the target to donor nerve diameter discrepancies, we chose to suture the donor nerves to the

341 motor entry point epimysially. In previous models, this approach led to reliable reinnervation of the

342 target muscle (Bergmeister et al., 2019).

343 Our behavioral observations indicate that the procedures did not cause extraordinary distress or pain

344 under adequate analgesia postoperatively. As early as one week after surgery, behavioral testing was

345 carried out in randomly selected individual animals, and all of them achieved the maximum score.

346 Likewise, after a 12-week regeneration period, all animals from both the control and the experimental

347 DNT group achieved the maximum score of Terzis grooming test (Inciong et al., 2000) (Video 1).

348 Hence, it seems that two motor nerves of different origin governing the same muscle did not hamper

349 activities of daily living. Additionally, no substantial pain or neuroma pain was evident. When

350 comparing the two procedures, it takes only marginally longer to perform the DNT, while no

351 additional physical stress or motor deficits were observed postoperatively.

352 The donor nerves reinnervated the target muscle within 12 weeks in all animals as indicated

353 macroscopically during dissection and by the fact that nerve crush or neurotomy induced 
354 fasciculations of the muscle (Videos 3 and 4). Likewise, intramuscular retrograde labeling showed

355 the uptake and transport of tracer dye into the motor neuron columns of the two transferred nerves.

356 Interestingly, after 12 weeks, muscle mass of the UN reinnervated muscles only recovered to 80.25

$357 \%$ of the contralateral side. This is in contrast with previous studies performed by authors of this

358 work (Bergmeister et al., 2019). A possible explanation for this mismatch is the difference of the

359 levels at which the UN was cut and transferred in the two studies. Unlike in the previous study where

360 the entire UN was transferred, here the UN was transferred at the wrist level. This may have caused

361 that the donor nerve was not able to fully regenerate the long head of the biceps due to the lower

362 motor axon numbers. Detailed analyses exist for humans, where the UN at wrist level only contains

$3631226 \pm 243$ motor axons compared to the entire UN $(2670 \pm 347)$ whereas the MCN contains $1601 \pm$

364164 (Gesslbauer et al., 2017). Considering that the muscle mass of double reinnervated muscles

365 regenerated to $98.83 \%$, it appears that the two donor nerves were better able to reinnervate and

366 adequately restore $24.72 \%$ more muscle mass than the SNT. This additionally indicates that both

367 SNT and DNT procedures were successful and that DNT with a high axonal load may lead to higher

368 muscle reinnervation and functional regeneration.

369 Previous findings (Bergmeister et al., 2019) reported neuroma formation at the insertion point

370 following nerve transfer. These consisted presumably mainly of sensory axons and the surplus of

371 motor neurons which was not able to innervate motor endplates. We did not observe neuroma

372 formation in this study and believe, that this is because the donor nerves comprised only few sensory

373 axons and the donor-to-recipient ratio of motor axons and targets was more balanced than in the

374 previous study, as mentioned above. Therefore, we assume that no fibers were lost at the insertion

375 site to the muscle, which may have formed a neuroma. Although the question of the optimal donor-

376 to-recipient ratio for optimal outcome remains unsolved, further investigations in this surgical model

377 are ongoing to answer this question and contribute to surgical refinement of nerve transfers. 
378 One potential limitation of this study is the use of the mixed UN containing both sensory and motor 379 nerve fibers. For better outcomes of surgical nerve transfers, "pure" motor nerves should be 380 preferred, such as the AIN used here, to avoid sensory to motor axon incongruence (Ray et al., 2016).

381 We decided to transfer the UN at a level, where it also contains sensory fibers of the superficial

382 branch because unlike in human, intraneural fascicular dissection to identify the two branches

383 proximal to Guyon's canal is impossible due to intermingling axons at the level of Guyon's canal.

384 Uncomplicated dissection, significant transfer leeway and the lack of a better alternatives made the

385 UN the best option.

386 The presented nerve transfer model finds broad application in many research fields. It offers the

387 possibility to investigate basic neurophysiology, but also clinical applications of surgical nerve

transfers for biological reconstruction and bionic reconstruction via targeted muscle reinnervation.

After amputation, targeted muscle reinnervation can create additional myosignals to improve basic prosthetic control. In TMR, neuromas within the stump are cut and the healthy fascicles are then

transferred to intact muscle segments, after denervation from their original innervation. EMG

technology can record and decipher neuronal signals from those reinnervated areas into signals for

prosthetic movement (Bergmeister et al., 2017;Muceli et al., 2019b;Salminger et al., 2019). The

biceps' long head is suitable to perform various EMG examinations, as we have previously shown

(Muceli et al., 2015), individual motor unit action potentials can potentially be decoded from such

signals as we have previously shown in SNT models (Muceli et al., 2019a).

398 In conclusion, this study demonstrated that a single target muscle can host two separate donor nerves.

399 Our results suggest that both the SNT and DNT models are suitable for common neurophysiological

400 examinations in peripheral nerve research. The concept of transferring multiple nerves to a single

401 target may improve muscle reinnervation, prosthetic interfacing, neuroma therapy or facilitate 
402 phantom limb pain management. Until first clinical applications can be translated, further research is

403 needed to fully understand the neurophysiological changes following multiple nerve transfers.

4045 Conflict of Interest

405 All authors declare that they have no competing interest. The ERC had no influence on the study.

\section{$406 \quad 6$ Author Contributions}

407 Conception and design: ML, JK, SM, JI, VT, CF, GL, OP, UM, DF, OCA and KDB. Analyses and

408 interpretation of data: ML, JK, SM, JI, VT, CF, GL, OP, UM, DF, OCA and KDB. Drafting of the

409 article: ML, SM, DF, OCA, and KDB. Critical revision for important intellectual content and final

410 approval of the version to be published: all authors.

\section{$411 \quad 7 \quad$ Funding}

412 This project has received funding from the European Research Council (ERC) under the European

413 Union's Horizon 2020 research and innovation program (grant agreement No 810346).

\section{$414 \quad 8 \quad$ Abbreviations}

415 AIN Anterior interosseus nerve

416 DNT Double nerve transfer

417 EMG Electromyography

418 MCN Musculocutaneous nerve

419 SNT Single nerve transfer

420 TMR Targeted muscle reinnervation

421 UN Ulnar nerve

4229 Acknowledgments 
423 We thank AM. Willensdorfer for her continuous technical assistance and A. Cserveny for his 424 admirable illustrations in this project. In addition, we thank Florian Frommlet for his statistical 425 analyses and expertise as a biostatistician.

\section{Data availability}

427 The following dataset was generated:

428 Luft et al. (2021), Muscle mass of the long head of the biceps following single and double nerve 429 transfer, Dryad, Dataset, https://doi.org/10.5061/dryad.3j9kd51jb

\section{References}

Aman, M., Sporer, M., Bergmeister, K., and Aszmann, O. (2019). Experimentelle Modelle für selektive Nerventransfers der oberen Extremität: Modellbeschreibung und neurophysiologische Effekte. Handchirurgie · Mikrochirurgie · Plastische Chirurgie 51, 319326.

Aszmann, O.C., Roche, A.D., Salminger, S., Paternostro-Sluga, T., Herceg, M., Sturma, A., Hofer, C., and Farina, D. (2015). Bionic reconstruction to restore hand function after brachial plexus injury: a case series of three patients. Lancet 385, 2183-2189.

Bergmeister, K.D., Aman, M., Muceli, S., Vujaklija, I., Manzano-Szalai, K., Unger, E., Byrne, R.A., Scheinecker, C., Riedl, O., Salminger, S., Frommlet, F., Borschel, G.H., Farina, D., and Aszmann, O.C. (2019). Peripheral nerve transfers change target muscle structure and function. Sci Adv 5, eaau2956.

Bergmeister, K.D., Aman, M., Riedl, O., Manzano-Szalai, K., Sporer, M.E., Salminger, S., and Aszmann, O.C. (2016). Experimental nerve transfer model in the rat forelimb. European Surgery 48, 334-341.

Bergmeister, K.D., Große-Hartlage, L., Daeschler, S.C., Rhodius, P., Böcker, A., Beyersdorff, M., Kern, A.O., Kneser, U., and Harhaus, L. (2020). Acute and long-term costs of 268 peripheral nerve injuries in the upper extremity. PLOS ONE 15, e0229530.

Bergmeister, K.D., Vujaklija, I., Muceli, S., Sturma, A., Hruby, L.A., Prahm, C., Riedl, O., Salminger, S., Manzano-Szalai, K., Aman, M., Russold, M.-F., Hofer, C., Principe, J., Farina, 

and Implantable Multichannel EMG Technology to Decode Spinal Motor Neuron Activity. Frontiers in Neuroscience 11.

Bertelli, J.A., and Mira, J.C. (1993). Behavioral evaluating methods in the objective clinical assessment of motor function after experimental brachial plexus reconstruction in the rat. $J$ Neurosci Methods 46, 203-208.

Bertelli, J.A., Mira, J.C., Pecot-Dechavassine, M., and Sebille, A. (1997). Selective motor hyperreinnervation using motor rootlet transfer: an experimental study in rat brachial plexus. J Neurosurg 87, 79-84.

Dumanian, G.A., Potter, B.K., Mioton, L.M., Ko, J.H., Cheesborough, J.E., Souza, J.M., Ertl, W.J., Tintle, S.M., Nanos, G.P., Valerio, I.L., Kuiken, T.A., Apkarian, A.V., Porter, K., and Jordan, S.W. (2019). Targeted Muscle Reinnervation Treats Neuroma and Phantom Pain in Major Limb Amputees: A Randomized Clinical Trial. Ann Surg 270, 238-246.

Farina, D., Vujaklija, I., Sartori, M., Kapelner, T., Negro, F., Jiang, N., Bergmeister, K., Andalib, A., Principe, J., and Aszmann, O.C. (2017). Man/machine interface based on the discharge timings of spinal motor neurons after targeted muscle reinnervation. Nature Biomedical Engineering 1, 0025.

Gesslbauer, B., Hruby, L.A., Roche, A.D., Farina, D., Blumer, R., and Aszmann, O.C. (2017). Axonal components of nerves innervating the human arm. Annals of Neurology 82, 396-408. Guillen, J. (2012). FELASA guidelines and recommendations. J Am Assoc Lab Anim Sci 51, 311321.

Hayashi, A., Moradzadeh, A., Hunter, D., Kawamura, D., Puppala, V., Tung, T., Mackinnon, S., and Myckatyn, T. (2007). Retrograde Labeling in Peripheral Nerve Research: It Is Not All Black and White. Journal of Reconstructive Microsurgery 23, 381-389.

Inciong, J.G., Marrocco, W.C., and Terzis, J.K. (2000). Efficacy of intervention strategies in a brachial plexus global avulsion model in the rat. Plast Reconstr Surg 105, 2059-2071.

Kapelner, T., Jiang, N., Holobar, A., Vujaklija, I., Roche, A.D., Farina, D., and Aszmann, O.C. (2016). Motor Unit Characteristics after Targeted Muscle Reinnervation. PLOS ONE 11, e0149772.

Kuiken, T.A., Childress, D.S., and Zev Rymer, W. (1995). The hyper-reinnervation of rat skeletal muscle. Brain Research 676, 113-123. 
Kuiken, T.A., Li, G., Lock, B.A., Lipschutz, R.D., Miller, L.A., Stubblefield, K.A., and Englehart, artificial arms. Jama 301, 619-628.

Mackinnon, S.E., and Novak, C.B. (1999). Nerve transfers. New options for reconstruction following nerve injury. Hand Clin 15, 643-666, ix.

Mioton, L.M., Dumanian, G.A., Shah, N., Qiu, C.S., Ertl, W.J., Potter, B.K., Souza, J.M., Valerio, I.L., Ko, J.H., and Jordan, S.W. (2020). Targeted Muscle Reinnervation Improves Residual Limb Pain, Phantom Limb Pain, and Limb Function: A Prospective Study of 33 Major Limb Amputees. Clin Orthop Relat Res 478, 2161-2167.

Muceli, S., Bergmeister, K.D., Hoffmann, K.P., Aman, M., Vukajlija, I., Aszmann, O.C., and Farina, D. (2019a). Decoding motor neuron activity from epimysial thin-film electrode recordings following targeted muscle reinnervation. J Neural Eng 16, 016010.

Muceli, S., Poppendieck, W., Hoffmann, K.P., Dosen, S., Benito-Leon, J., Barroso, F.O., Pons, J.L., and Farina, D. (2019b). A thin-film multichannel electrode for muscle recording and stimulation in neuroprosthetics applications. J Neural Eng 16, 026035.

Muceli, S., Poppendieck, W., Negro, F., Yoshida, K., Hoffmann, K.P., Butler, J.E., Gandevia, S.C., and Farina, D. (2015). Accurate and representative decoding of the neural drive to muscles in humans with multi-channel intramuscular thin-film electrodes. J Physiol 593, 3789-3804.

Oberlin, C., Béal, D., Leechavengvongs, S., Salon, A., Dauge, M.C., and Sarcy, J.J. (1994). Nerve transfer to biceps muscle using a part of ulnar nerve for C5-C6 avulsion of the brachial plexus: anatomical study and report of four cases. J Hand Surg Am 19, 232-237.

Percie Du Sert, N., Hurst, V., Ahluwalia, A., Alam, S., Avey, M.T., Baker, M., Browne, W.J., Clark, A., Cuthill, I.C., Dirnagl, U., Emerson, M., Garner, P., Holgate, S.T., Howells, D.W., Karp, N.A., Lazic, S.E., Lidster, K., Maccallum, C.J., Macleod, M., Pearl, E.J., Petersen, O.H., Rawle, F., Reynolds, P., Rooney, K., Sena, E.S., Silberberg, S.D., Steckler, T., and Würbel, H. (2020). The ARRIVE guidelines 2.0: Updated guidelines for reporting animal research. PLOS Biology 18, e3000410.

Ray, W.Z., Chang, J., Hawasli, A., Wilson, T.J., and Yang, L. (2016). Motor Nerve Transfers. Neurosurgery 78, 1-26.

Salminger, S., Sturma, A., Hofer, C., Evangelista, M., Perrin, M., Bergmeister, K.D., Roche, A.D., Hasenoehrl, T., Dietl, H., Farina, D., and Aszmann, O.C. (2019). Long-term implant of intramuscular sensors and nerve transfers for wireless control of robotic arms in above-elbow amputees. Science Robotics 4, eaaw6306. 
515 Scholz, T., Krichevsky, A., Sumarto, A., Jaffurs, D., Wirth, G., Paydar, K., and Evans, G. (2009).

516 Peripheral Nerve Injuries: An International Survey of Current Treatments and Future

517 Perspectives. 25, 339-344.

518 Terzis, J.K., and Papakonstantinou, K.C. (2000). The surgical treatment of brachial plexus injuries in 519 adults. Plast Reconstr Surg 106, 1097-1122; quiz 1123-1094.

520 Van Zyl, N., Hill, B., Cooper, C., Hahn, J., and Galea, M.P. (2019). Expanding traditional tendon-

521 based techniques with nerve transfers for the restoration of upper limb function in tetraplegia:

522 a prospective case series. The Lancet 394, 565-575.

\section{$523 \quad 12$ Rich Media}

524 Video 1: Grooming behavior 12 weeks following double nerve transfer in the right upper limb.

525 Video 2: Muscle response upon crushing the motor branch of the long head of the biceps.

526 Video 3: Muscle response upon ulnar nerve crush following double nerve transfer.

527 Video 4: Muscle response upon anterior interosseus nerve crush following double nerve transfer. 